\title{
Selected papers in nonlinear dynamics in dissipative and discrete optical systems
}

\author{
F. Lederer $\cdot$ S. Nolte $\cdot$ A. Tünnermann
}

Published online: 29 July 2011

(C) Springer-Verlag 2011

In recent years much attention has been devoted to the study of intrinsic localization of light, i.e. the formation of solitons, in realistic nonlinear systems. This concerns essentially settings where the energy exchange with the environment (dissipative systems) and the noninstantaneous nonlinear response of the respective nonlinear material are taken into account. Another interesting aspect in nonlinear physics is the role of discreteness. Energy exchange (or dissipation) and the noninstantaneous response affect to a large extent the action of the nonlinearity, whereas discreteness offers new opportunities to control the linear properties of the system. Consequently, all these effects and their interplay will evoke a plethora of new soliton solutions. This special issue of Applied Physics B contains selected results of the DFG Research Unit 512 'Nonlinear spatio-temporal dynamics in dissipative and discrete optical systems' together with guest contributions of Stefano Longhi, Politecnico di Milano, and the group of Zhigang Chen, San Francisco State University. The subject looked at by the Research Unit was in its infancy when we started our project. Meanwhile, it has attracted a great deal of interest and lies well in the mainstream of contemporary research in optics.

In order to identify the wealth of the underlying physics, we looked at various optical systems spanning from fairly simple ones such as passive and active optical fibers and media exhibiting a second- or third-order nonlinearity to more

\section{F. Lederer}

Institute of Condensed Matter Theory and Solid State Optics, Friedrich-Schiller-Universität Jena, Max-Wien-Platz 1, 07743 Jena, Germany

S. Nolte $(\bowtie) \cdot$ A. Tünnermann Institute of Applied Physics, Friedrich-Schiller-Universität Jena, Max-Wien-Platz 1, 07743 Jena, Germany

e-mail: stefan.nolte@uni-jena.de complex ones such as linear and nonlinear photonic lattices (fiber arrays or optically generated arrays in fused silica) and isolated as well as coupled ensembles of microstructured resonators. The contributions cover very fundamental issues such as the simulation of quantum phenomena in purely classical systems, vortices in ultra-short laser pulses, photonic Bloch oscillations in the spectral domain, and the formation of cavity solitons in all-photonic crystal resonators to issues with large application potential such as e.g. the complete understanding and control of pulse dynamics and interactions in mode-locked fiber lasers.

The role of discreteness in optics has been studied in various settings. Stefano Longhi discusses the unique possibilities offered by light transport in engineered photonic lattices and Bragg grating structures to simulate in a purely classical system the optical analogues of a wide variety of quantum phenomena such as e.g. the Zitterbewegung of a relativistic electron, Klein tunneling, vacuum decay and pair production, and the Dirac oscillator. Matthias Heinrich et al. prove that two-dimensional femtosecond laser-written arrays in fused silica represent a versatile and unrivaled test bed for studying both linear and nonlinear discrete optical effects such as e.g. the formation of vector solitons, solitons in superlattices, and the controllable reflection/transmission properties of light at these solitons. Another option to fabricate highly accurate photonic lattices is discussed by Ulrich Röpcke et al. They show that arrays of closely spaced optical fibers exhibit an extraordinarily perfect discrete diffraction pattern and are well suited for the first experimental demonstration of stable spatio-temporal light localization, i.e. the excitation of discrete-continuous light bullets. Light propagation in lithium niobate arrays which exhibit strong second-order nonlinearity is experimentally investigated by Frank Setzpfand et al., where the excitation of higher order bands by the second-harmonic field is taken into account. 
Thus, novel effects can be studied such as e.g. the interaction of a single fundamental field with a few second-order harmonic fields which may lead to new scenarios in discrete soliton formation. Christoph Bersch et al. pursue a very exciting analogy. Using a simple optical fiber, they generate a time-periodic potential, which is the temporal analogue to a photonic lattice, with a superimposed linear variation by exploiting both electro- and acousto-optical modulation. It is then shown that a short pulse traversing this fiber exhibits temporal Bloch oscillations. Moreover, superposition in a highly nonlinear fiber leads to Bloch oscillations in the frequency domain. A quite different nonlinear discrete system is looked at by Carsten Schmidt et al. They study the linear and temperature-induced nonlinear behavior of coupled silica microresonators.

Light localization in nonconservative systems, i.e. the formation of temporal and spatial dissipative solitons, is studied in another series of contributions. It is shown theoretically by Aleksandr Zavyalov et al. and experimentally and numerically by Martin Baumgartl et al. that the pulse dynamics in mode-locked fiber lasers, consisting essentially of a nonlinear fiber with gain and loss, a saturable absorber, and some dispersion-controlling and coupler elements, may be explained in the framework of dissipative solitons. The latter work identifies several stable operation regimes at positive net cavity dispersion. In particular, pulse formation and evolution in the chirped-pulse regime at highly positive cavity dispersion is discussed. The former contribution fo- cuses on the formation of bound states or so-called soliton molecules. The interesting finding is that these molecules become stable if the response of the saturable absorber is fast, but not instantaneous. Spatial dissipative solitons, i.e. Bloch cavity solitons, with novel properties, such as e.g. a controllable and very small transverse size, have been found theoretically by Oleg Egorov et al. in an all-photonic crystal resonator where the dispersion can be tailored by the geometry of the photonic crystal.

The possibility of creating channels for light propagation by exploiting the photorefractive effect in lithium niobate has been experimentally studied by Oleg Kashin et al. Peng Zhang et al. also studied photonic lattices optically induced in photorefractive materials. The generated threedimensional structures enabled new opportunities for controlling the flow of light. By tuning the lattice parameters, various unusual behaviors of beam propagation could be demonstrated, including enhanced discrete diffraction, coherent destruction of tunneling, anomalous diffraction, and negative refraction.

Optical vortices in a Kerr medium nested in a broadband femtosecond laser beam were investigated by Peter Hansinger et al. It has been shown that vortex-vortex interactions do not depend on the sign of the nonlinearity and that regimes of stable multiple filamentation exist.

Acknowledgements We would like to thank the German Science Foundation (DFG) for funding this exciting research. 